\title{
Effect of Mixed Spikes on Different Types of Complex Waves
}

\author{
A.N.M. Rezaul Karim \\ Correspondence: Department of Computer Science \& Engineering, International Islamic University Chittagong, Kumira, \\ Sitakunda, Chittagong-4318, Bangladesh. E-mail: zakianaser@yahoo.com
}

Received: April 1, 2019 Accepted: October 1,2019 Online Published: November 24, 2019

doi:10.5539/jmr.v11n6p70

URL: https://doi.org/10.5539/jmr.v11n6p70

\begin{abstract}
This article treats analytically. This paper presents a novel approach to complex waves. This article outlines the understanding of the various effects of spike representations used to make models of the predictive variable effects of the second-order portion of power while revealing the relationship between the time series segments that are recorded from a single unit. MATLAB has been used to show the effects of mixed spikes in graphs. The resulting power portion has varied representation effects in which both the random and fixed effects are expressed as functions of the frequency domain.
\end{abstract}

Keywords: spectrum, Fourier series, mixed effects

\section{Introduction}

Various sinusoid waveforms can be put together to make up a single complex waveform, a Fourier series. The sinusoids are of different frequencies, phases and amplitudes. The magnitude, frequency and phase descriptions of the sinusoid waveforms are the signal spectrum (portion); by comparison the light spectrum.

The Fourier series is a periodic function used to express the frequency domains of the related sinusoids. This frequency domain is represented graphically as the series of spikes that are determined by the original function's period and its harmonically related sinusoids. The Fourier coefficients represent the spikes' magnitude. The series of these components is the wave. The analysis of the time-domain waveform to determine its spectrum is referred to as spectrum analysis of complex wave (James, 1999)

In several experiments, the time-series data is obtained from different units while the segments of these time-series are obtained from the same entity. The paper outlines the mixed-effects representation of the spectrum that can be used to create a model of the second-order spectral power covariates whilst explaining the reliable correlations of the above collected time-series segments that are obtained from a single unit. The log-spectrum contains the mixed-effects functional representation where both the random and fixed effects are considered as functions in the same frequency domain.

In a biomedical experiment, the data about the time-series are commonly collected from several subjects and used as the basic unit to analyze the design covariate effects. Among the studies are the multiple segments of the time-series that are obtained from a single unit that can be potentially correlated.

Diggle\&Wasel (1997) introduces an independent model used to collects and replicate the time series unit-specific spectra via log-linear effects. A review by Iannaccone \& Coles (2001) provides a generalization of the model through the estimation of the nonparametric spline of these fixed effects. There also exist tree-structures methods of wavelet purposely for evaluation of the replicated time-series spectra. The models are exclusively designed to collectively analyze the time series using a simple while and structure in which the mutually independent series are mutually related (Freyermuth et al. 2010). The novel contribution of the article outlines the mixed-effects representation of the Cramer for these collective models of the stationery time-series which exploits which uses a flexible linear model for both the random and fixed effects in which many designs are handled by specific design metrics (Laird \& Ware, 1982). The model takes note the existing correlations of the time series spectra segments of a common unit.

A number of researchers including Zhang \& Chen (2007), Rice \& Wu (2001), Morris \& Carroll (2006), Guo (2002), Brumback\& Rice (1998), Wu \& Zhang (2002), Staniswalis\& Lee (1998), and Rice \& Silverman (1991)have focused on study of the fixed and random functional mixed-effect models.

Our proposed model assumes the random effects to be Gaussian but does not make assumptions on the log-spectral random effects distribution. We suggest an algorithm which begins by estimating the initial spline for the fixed effects of the log-spectral. The initial spline estimator is obtained through the approximation of the penalized sum-of-squares' minimizer that does not take into account the log-spectral units that are correlated and be seen as the extension of the 
estimators. The log-spectra's first two moments can be used to find the estimate the random effects of the unit-specific moments using the plug-in estimates obtained by unbiased best linear points. The fixed effects are again estimated through approximation of the minimizer of penalized sum-of-squares by doing away with the estimated effects that are unit-specific and related to the log-period grams.

The Fourier series breaks down a signal into multiple sines and cosines having varying frequencies. Also, the Fourier representation is periodic; the beginning of the cycle equals the (i.ef $(t=0)=f(t=1)$ ). When the procedure is reversed, the periodic signal can be formed by superimposing the sinusoidal and the co-sinusoidal waves.

Mathematically, the Fourier series can be represented by the periodic signal $\mathbf{f}(\mathbf{t})$ as follows:

Let a complex wave (Fourier series) $f(t)$ represent a periodic with period $T$

$$
f(t)=\frac{a_{0}}{2}+\sum_{n=1}^{\infty}\left(a_{n} \cos (n \omega t)+b_{n} \sin (n \omega t)\right)
$$

- $\frac{a_{0}}{2} \rightarrow \mathrm{DC}$ value

- The $a_{n}$ holds the amplitudes of the cosine wave

- The $b_{n}$ holds the amplitudes of the sine wave

- $\quad n \rightarrow$ harmonic

- $\omega_{0} \rightarrow$ Fundamental frequency, $\left[\because \omega_{0}=\frac{2 \pi}{T}\right]$

- $\quad T \rightarrow$ Period of $f(t)$

$$
\begin{aligned}
& \underset{\text { Complex wave }}{f(t)} \approx \frac{a_{0}}{2}+\underbrace{\sum_{n=1}^{\infty}\left(a_{n} \cos \left(n \frac{2 \pi}{T} t\right)+b_{n} \sin \left(n \frac{2 \pi}{T} t\right)\right)}_{\text {DC value }} \\
& f(t) \sim \sum_{n=0}^{\infty} a_{n} \cos \left(n \frac{2 \pi}{T} t\right)+\sum_{n=1}^{\infty} b_{n} \sin \left(n \frac{2 \pi}{T} t\right) \\
& f(t) \sim \sum_{n=0}^{\infty} R_{n} \sin \left(n \frac{2 \pi}{T} t+\theta_{n}\right)
\end{aligned}
$$

The modulation of the Fourier series amplitude improves the localization of the frequency of the Fourier series. This modulation makes the amplitude $R_{n}$, the equation (ii) is called amplitude-modulated Fourier series (AMFS).

The amplitude plot $R_{n}=\sqrt{a_{n}^{2}+b_{n}^{2}}$ against the frequency $\omega$ (use the function $R_{n}=\sqrt{a_{n}^{2}+b_{n}^{2}}$. For each of the harmonics $(n=0,1,2,3, \cdots)$, a point is plotted and therefore we have to know $n$ and $\omega$ relate to each other [Glyn James, 1999]. The phase plot $\theta_{n}=\tan ^{-1}\left(\frac{a_{n}}{b_{n}}\right)$ against the (radian) frequency $\omega$ and

$$
\begin{gathered}
a_{0}=\frac{1}{\pi} \int_{-\pi}^{\pi} f(t) d t \\
a_{n}=\frac{1}{\pi} \int_{-\pi}^{\pi} f(t) \cos (n t) d t
\end{gathered}
$$




$$
b_{n}=\frac{1}{\pi} \int_{-\pi}^{\pi} f(t) \sin (n t) d t
$$

\section{Model: Mixed Spectral Representation}

We introduce the mixed-effects of $2^{\text {nd }}$ order spectral representation is

$$
z(t)=f(t) * s(t))=\sum_{n=0}^{\infty} \sum_{m=0}^{\infty} R_{(n, m)}\left\{\sin \left(n \frac{2 \pi}{T} t+\theta_{n}\right)\right\}\left\{\sin \left(m \frac{2 \pi}{T} t+\theta_{m}\right)\right\}
$$

Where $\mathrm{f}(\mathrm{t})$ and s ( $\mathrm{t}$ ) are two complex waves and Period $T=2 L$

Then,

$$
\therefore a_{0}^{2}=\frac{1}{L} \int_{-L}^{L} f(t) d t * \frac{1}{L} \int_{-L}^{L} s(t) d t
$$

[Here, $\omega=\frac{2 \pi}{T}=\frac{2 \pi}{2 L}=\frac{\pi}{L}$ ]

$$
\begin{aligned}
& a_{n}{ }^{2}=\frac{1}{L} \int_{-L}^{L} f(t) \cos \left(n \frac{\pi}{L} t\right) d t * \frac{1}{L} \int_{-L}^{L} s(t) \cos \left(n \frac{\pi}{L} t\right) d t \\
& b_{n}^{2}=\frac{1}{L} \int_{-L}^{L} f(t) \sin \left(n \frac{\pi}{L} t\right) d t * \frac{1}{L} \int_{-L}^{L} s(t) \sin \left(n \frac{\pi}{L} t\right) d t
\end{aligned}
$$

The following analog spectrum analyzer should be considered in order to have a good understanding of the Fourier analysis:

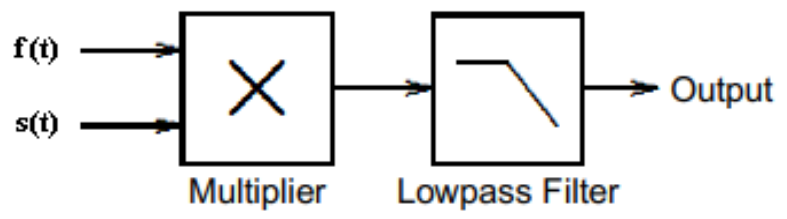

Figure 1. Mixed Effect analyzer for spectrum

The unknown signal $f_{t}$; which contains multiple components at different frequencies is inputted into one of the multiplier. The variable-frequency sine waveform, the frequency $s_{t}$ is inputted into another multiplier. The multiplier output, computed as the product of the two signals is set as the low pass filter and then to the voltmeter.

When performing the spectrum analysis, the signal sis slowly taken through the frequencies to be used for the analysis. The product of the two frequencies in the multiplier produces new frequencies at the output; one of the frequencies generated at the sum $\mathrm{f}+\mathrm{s}$, and another generated at the difference $\mathrm{f}$-s. The sum frequency is rejected by the low pass filter. While the frequencies approach one another, the difference frequency, $f-s$ approaches zero. At some point, the difference drops within the low pass filter as an AC signal measured using the voltmeter. If $\mathrm{f}$ coincides with $\mathrm{s}$, the low pass filter output becomes a Dc signal that is proportional to their component magnitudes.

The analysis of the spectrum can consequently be performed by doing away with the oscillator $\mathrm{s}$ and recording both the signal amplitude and frequency each time it appears at the low pass filter output.

\section{Estimation}

\subsection{Example}

Suppose a complex wave with a period $T=2 L=2 \pi$ whose equation is

$$
\underset{\text { Complex wave }}{f(t)}=\underset{D C \text { value }}{0}+\underbrace{\sum_{n=1}^{\infty} \frac{2}{n \pi}(1-\cos n \pi) \sin n t}_{\text {AC value }}
$$




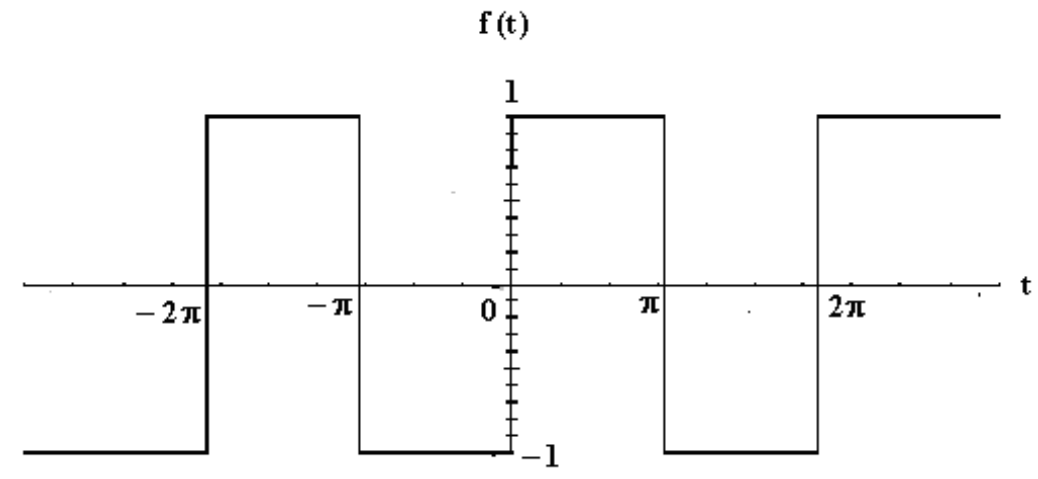

Figure 2. Complex wave of $f(t)$ in time domain

3.1.1 Frequency Spectrum of $f(t)$

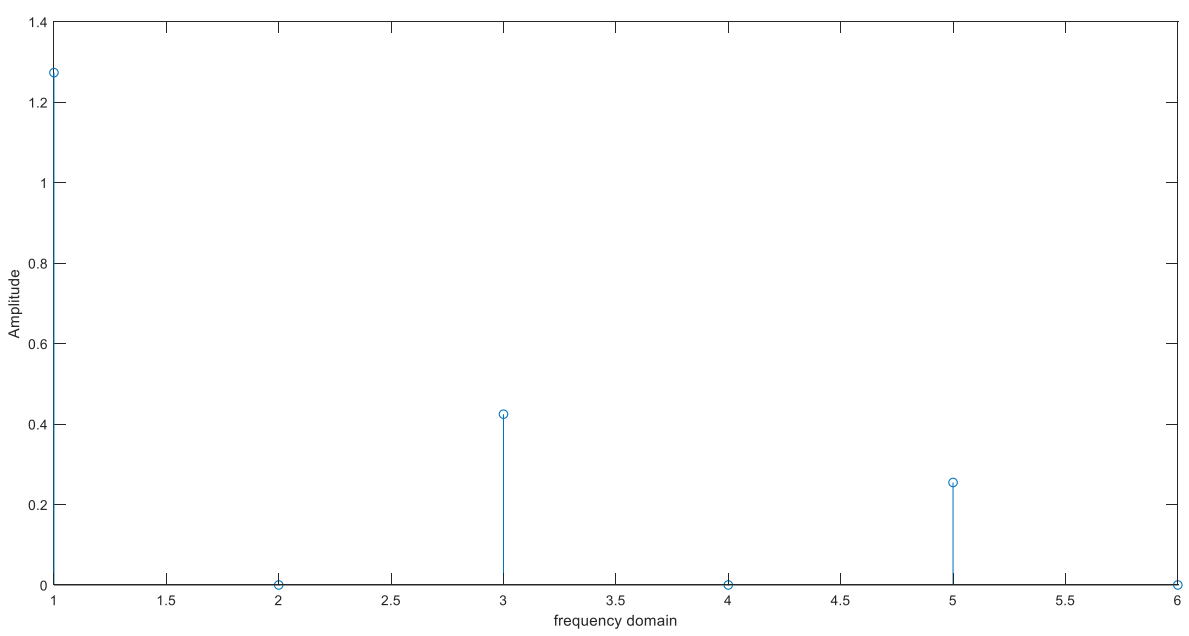

Figure 3. Spectrum of $\mathrm{f}(\mathrm{t})$ in Frequency domain

3.2 Suppose an Another Complex Wave With a Period $T=2 L=2 \pi$ Whose Equation Is

$$
s(t)=\underbrace{0}_{D C \text { value }}+\underbrace{-2 \sum_{n=1}^{\infty} \frac{1}{n} \cos n \pi \cdot \sin n t}_{A C \text { value }}
$$

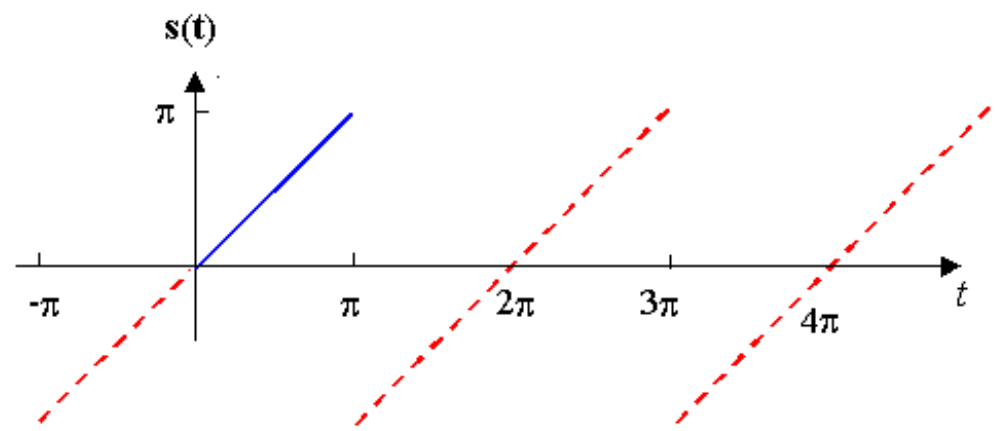

Figure 4.Complex wave of $s(t)$ in time domain 


\subsubsection{Frequency Spectrum of $s(\mathrm{t})$}

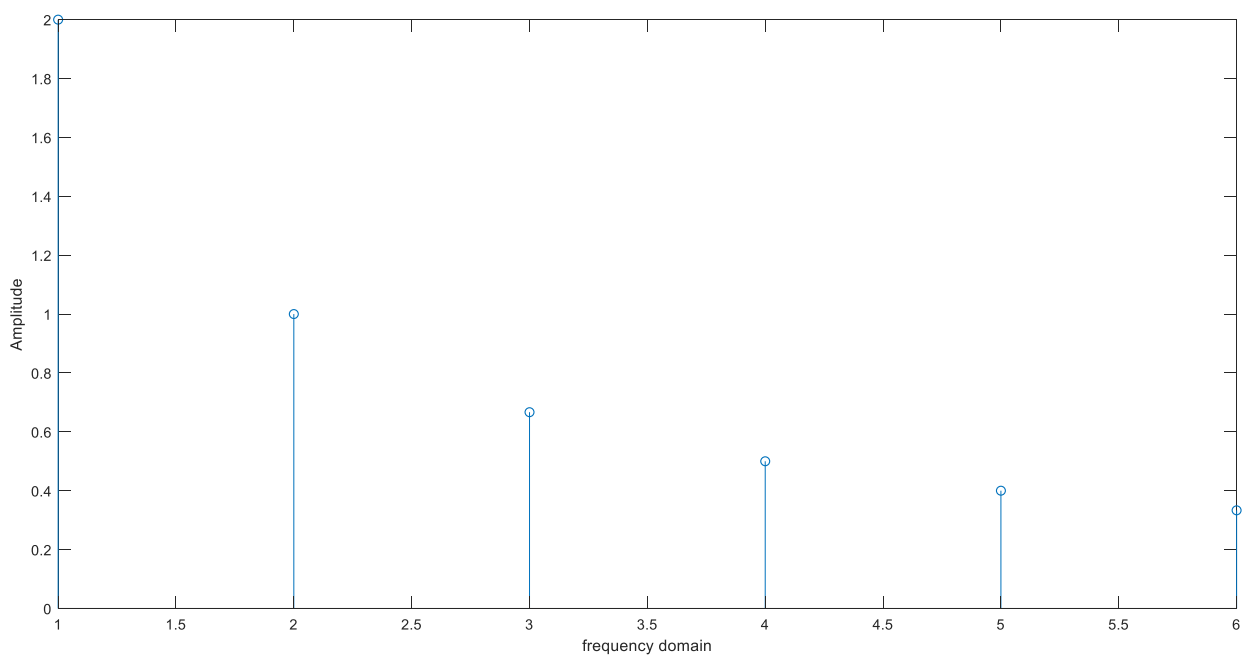

Figure 5. Spectrum of $s(t)$ in Frequency domain

\subsection{Fixed Effect}

In order to find out the empirical performance according to the proposed estimation of this article, simulation studies were carried out in comparison to the performance of other three different systems. The estimates of the fixed-effects are then ordinarily obtained from the least-squares at the different frequencies. The evaluation of the steps of performance is done through square error estimation from the plotted-spectral against the determined Fourier frequencies, all of estimate, plotted-spectral estimated and the replicate-specific of the kernel just as shown below.

This finding generally implies that efficiency of the estimations which is also a relative gain is inversely proportional to the ration of $\mathrm{T}$ to $\mathrm{N}$. The findings are due to the fact that the above best linear estimates of the assessment of a log-spectrum demands a proper estimation of the kernel's covariance which relies of $\mathrm{N}$. on the other hand, the pre-smoothing data performance of the estimates of the log-spectrum depends only on $\mathrm{T}$.

\section{Simulations}

$$
\begin{gathered}
a_{n}^{2}=0 \\
b_{n}^{2}=\frac{2}{n \pi}(1-\cos n \pi) * \frac{-2}{n} \cos n \pi \\
R_{n}=\sqrt{a_{n}^{2}+b_{n}^{2}}
\end{gathered}
$$

The value of $R_{n}=\sqrt{a_{n}^{2}+b_{n}^{2}}$ 


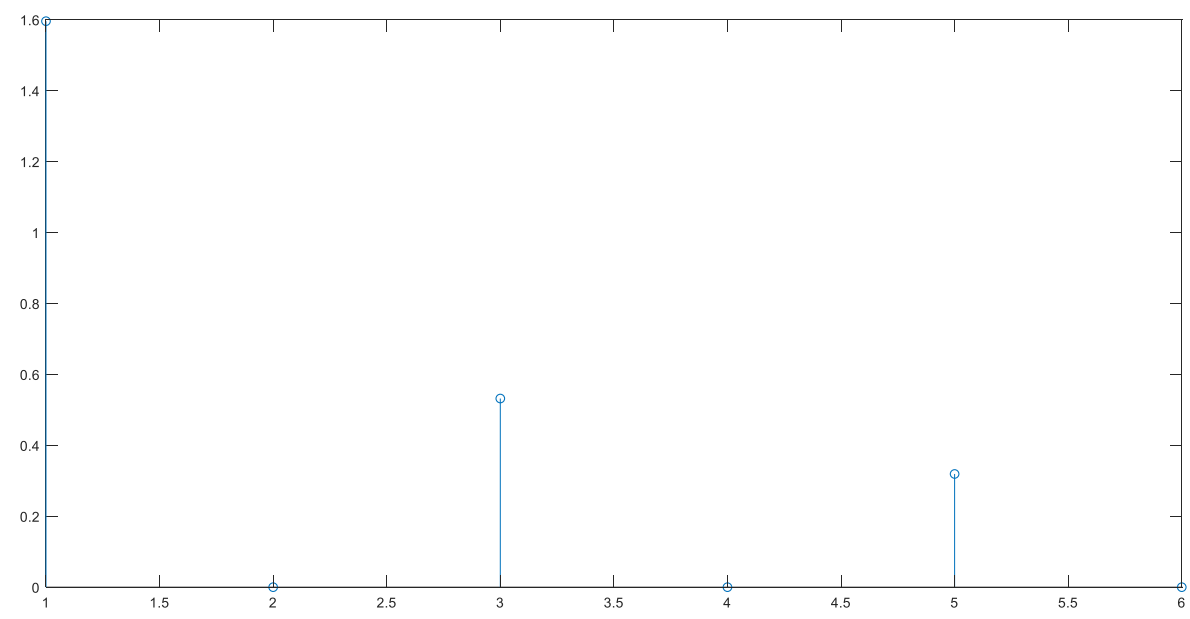

Figure 6. Frequency spectrum of $\mathrm{z}(\mathrm{t})$

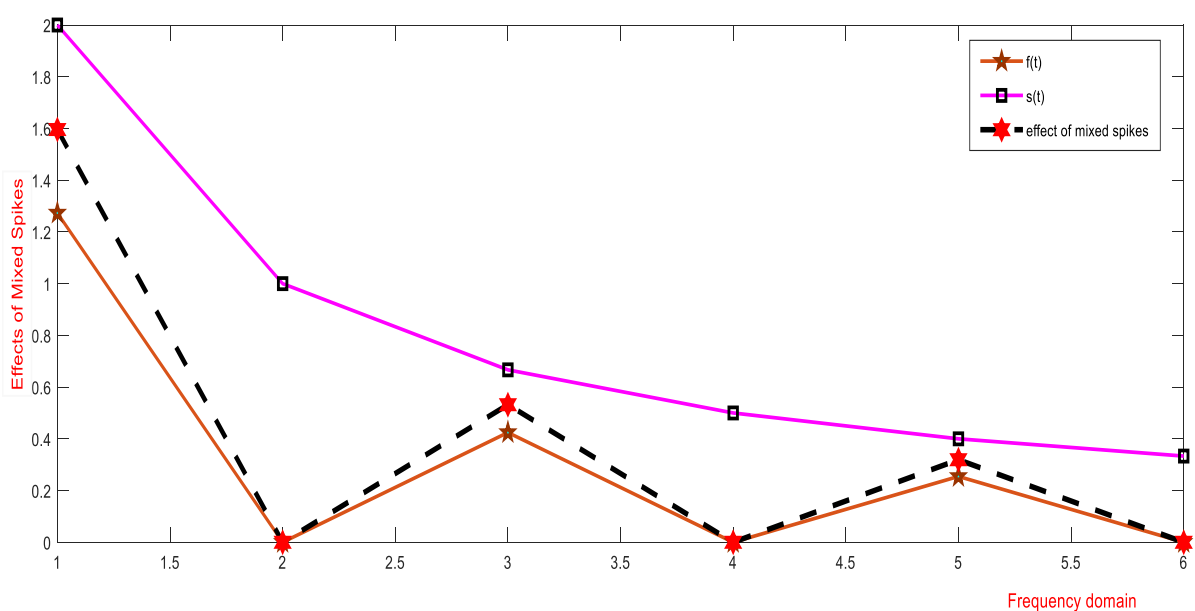

Figure 7. Effects of mixed spikes apply to different types of complex waves

\section{Discussion}

This article introduced an estimation procedure and model that provides for the important tools for the analysis of the time series collection from designs implementable through multiple directions to influence the other dominant settings. In this article, our focus was on estimation-based for the first two log-spectra moments. We further extend to the Whittle-likelihood-based interference procedure. Moreover, a number of the applications imply the replicated break-down of the flexible time series. Guo et al. (2003) employ the use of the tensor-product which is also incorporated into our suggested procedure to help in the time series spectrum analysis. To prove the usefulness of the application of this model, we incorporate iterative estimation procedure to make use of the tools that can take care of the local properties including the wavelet.

\section{Conclusion}

In every systems of communication, the center of interest is the sequential transmission of the pulse shape, that is, the differing amplitudes from every pulse rather that the periodically repeated pulse. It is therefore necessary to consider the present frequencies that represent a single pulse of the duration $\tau$ whose power is slowly decaying.

\section{References}

Brumback, B. A., \& Rice, J. A. (1998). Smoothing spline models for the analysis of nested and crossed samples of curves. J. Am. Statist. Assoc., 93, 961-94. https://doi.org/10.2307/2669837

Diggle, P. J., \& Wasel, I. (1997).Spectral analysis of replicated biomedical time series. Appl. Statist, 46, 37-71. https://doi.org/10.1111/1467-9876.00047

Freyermuth, J. M., Ombao, H., \&Vonsachs, R. (2010). Tree-structured wavelet estimation in a mixed effects model for spectra of replicated time series. J. Am. Statist. Assoc., 105, 634-46. https://doi.org/10.1198/jasa.2010.tm09132 
Guo, W. (2002). Functional mixed effects models. Biometrics, 58, 121-8. https://doi.org/10.1111/j.0006-341X.2002.00121.x

Guo, W., Dai, M., Ombao, H., \&Vonsachs, R. (2003). Smoothing spline anova for time-dependent spectral analysis. $J$. Am. Statist. Assoc., 98, 643-5. https://doi.org/10.1198/016214503000000549

Iannaccone, R., \& Coles, S. (2001). Semi parametric models and inference for biomedical time series with extravariation. Biostatistics, 2, 261-76. https://doi.org/10.1093/biostatistics/2.3.261

James, G. (1999). Advanced Modern Engineering Mathematics (2nd ed.), 338.

Laird, N. M., \& Ware, J. H. (1982). Random-effects models for longitudinal data. Biometrics, 38, 963-74. https://doi.org/10.2307/2529876

Morris, J. S., \& Carroll, R. J. (2006).Wavelet-based functional mixed models. J. R. Statist. Soc., 68, 179-99. https://doi.org/10.1111/j.1467-9868.2006.00539.x

Rice, J. A., \& Silverman, B. W. (1991).Estimating the mean and covariance structure nonparametrically when the data are curves. J. R. Statist. Soc., 53, 233-43. https://doi.org/10.1111/j.2517-6161.1991.tb01821.x

Rice, J. A., \& Wu, C. O. (2001). Nonparametric mixed effects models for unequally sampled noisy curves. Biometrics, 57, 253-9. https://doi.org/10.1111/j.0006-341X.2001.00253.x

Staniswalis, J. G., \& Lee, J. J. (1998).Nonparametric regression analysis of longitudinal data.J. Am. Statist. Assoc., 93, 1403-18. https://doi.org/10.1080/01621459.1998.10473801

WU, H., \& Zhang, J. T. (2002). Local polynomial mixed-effects models for longitudinal data. J. Am. Statist. Assoc., 97, 883-97. https://doi.org/10.1198/016214502388618672

Zhang, J. T., \& Chen, J. (2007). Statistical inferences for functional data. Ann. Statist., 35, 1052-79. https://doi.org/10.1214/009053606000001505

\section{Copyrights}

Copyright for this articleis retained by the author(s), with first publication rights granted to the journal.

This is an open-access article distributed under the terms and conditions of the CreativeCommons Attribution license (http://creativecommons.org/licenses/by/4.0/). 